\title{
Die Wahl der Implantate ist Aufgabe der Ärztinnen und Ärzte
}

\section{Josef E. Brandenberg}

Dr. med., Facharzt für Orthopädische Chirurgie und Traumatologie, FMH, Ombudsmann der fmCh und von Swiss Orthopaedics

\begin{abstract}
Wenn die Wahl der Implantate ausschliesslich aus ökonomischer Sicht erfolgt, besteht die Gefahr der Qualitätseinbusse, insbesondere, wenn Zusatzleistungen wie Lieferservice rund um die Uhr, Aktualisierung des Instrumentariums, Support der Firmen bei Revisionsoperationen wegfallen. Auch unter SwissDRG müssen die Operateure die Implantate wählen. Die Aushandlung der Lieferbedingungen und Preise ist Sache der Spitalverwaltung.
\end{abstract}

\section{Einleitung}

Ein neues Phänomen prägte den Kongress der American Academy of Orthopaedic Surgeons AAOS im Jahr 2007. In San Francisco waren alle Taxis, LitfassSäulen und die legendären Cable Cars grossflächig dekoriert mit Reklamen verschiedener Prothesenfirmen. "No more pain - Hip Prosthesis WX», «The step towards the future - Knee Replacement YZ» usw.

Evolution problématique dans le domaine de l'endoprothèse

Depuis l'introduction du système de forfaits par cas SwissDRG, la tendance est à la réduction des coûts de traitement, une situation qui n'épargne pas les implants. Si le produit le plus cher n'est pas toujours le meilleur, un choix fondé uniquement sur des critères économiques et sur le prix risque cependant de prétériter la qualité, notamment lorsque certains services annexes ne sont plus inclus dans le prix (disponibilité, actualisation du matériel, assistance du fabriquant pour les opérations de révision, etc.).

Un autre danger réside dans les changements fréquents de produit. En effet, chaque changement suppose une phase d'apprentissage de la part de l'opérateur, ce qui augmente la durée de l'opération, le taux d'erreur et le risque de complications. II importe donc d'exiger que les opérateurs puissent, également sous SwissDRG, choisir eux-mêmes les implants et qu'ils s'en tiennent à ce choix aussi longtemps que possible, dans la mesure où les implants choisis sont de bonne qualité. Les négociations autour des délais de livraison et des prix relèvent quant à elles de l'administration de l'hôpital. Les avantages financiers de même que les assistances opératoires mises gratuitement à disposition par les fabricants sont à proscrire. A cet égard, il convient de se référer aux directives de l'Académie suisse des sciences médicales sur la collaboration corps médicalindustrie et celles de Swiss Orthopaedics sur le choix des implants sous SwissDRG.
Meine US-amerikanischen Freunde bestätigten: Solche Reklamen werden auch im Fernsehen gezeigt. Der irritierte Gast aus Europa fragte sich, was das soll. Schliesslich wählt doch nicht der Patient - pardon der Endverbraucher - seine Prothese, sondern der Arzt. US-amerikanische Firmenvertreter erklärten mir, dieses damals neue, zukunftsweisende Marketing - «Pull and Push» genannt - funktioniere wie folgt: Man ziehe den Konsumenten ans Produkt heran und dieser übe dann Druck auf den Operateur aus, damit er dieses implantiere. An sich logisch und ökonomisch konsequent. Der Patient ist letztlich Eigentümer des Implantates und kann als Kunde frei-marktwirtschaftlich das Konsumgut selber auswählen. Dass dieses Konzept funktioniert, mag sich durch die Tatsache erklären, dass in den USA 80\% der Operateure weniger als 20 Hüftprothesen pro Jahr implantieren. Da schwenkt sicher noch der eine oder andere Operateur auf die Wünsche der Patienten ein, ungeachtet der Qualitätseinbusse. Doch darüber weiter unten.

\section{Marketing in der Schweiz}

Zurück in die Schweiz. 2007 war die Welt noch in Ordnung. Aber war sie es wirklich? Und ist sie es heute noch? Vorab ist klar: In der Schweiz ist das «Pull and Push»-Marketing nicht erlaubt. Bis vor kurzem fragten Patientinnen und Patienten auch selten nach der Herkunft des Implantates. Man vertraute dem Operateur. Einzig nach einschlägigen Gesundheitssendungen im Fernsehen wurde das gerade aktuell «neueste, sensationelle Verfahren» oder «die ultimativ beste Implantate-Neuheit» zum Thema. Viele 
Ärzte, nicht die Industrie, verbreiteten vor laufender Kamera diese reisserischen Werbebotschaften. Die meisten Firmen unterstützten allerdings ihre ärztlichen Kunden bei solchen Werbe-Feldzügen aus dem Hintergrund. "Pull and Push» made in Switzerland. Und die Spitalträger taten dasselbe - unter unmissverständlicher Nennung des Namens des Spitals oder der Klinik. Schliesslich waren es schon immer die Spitalträger, die die Produkte einkauften, lagerten und den Kostenträgern in Rechnung stellten. Im Gespräch mit Firmenvertretern klang es zwar so: «Wir sind im Spital X drin» oder die «Klinik Y haben wir an die Konkurrenz verloren». Die eigentlichen Entscheidungsträger waren aber - bis vor kurzem - die Ärzte.

\section{Auswahlkriterien}

Doch nach welchen Kriterien wurde und wird die Implantatwahl getroffen? Um es vorweg zu nehmen: Der Preis war bis vor kurzem kein Thema. Es existiert keine wissenschaftliche Studie, auch keine Marktanalyse, die der Frage nach den Auswahlkriterien nachgegangen wäre. Es ist anzunehmen, dass die gemachten Erfahrungen während der Weiterbildung zum Facharzt prägend waren. In der Hüfte-Endoprothetik sind die Anhänger einer metaphysären

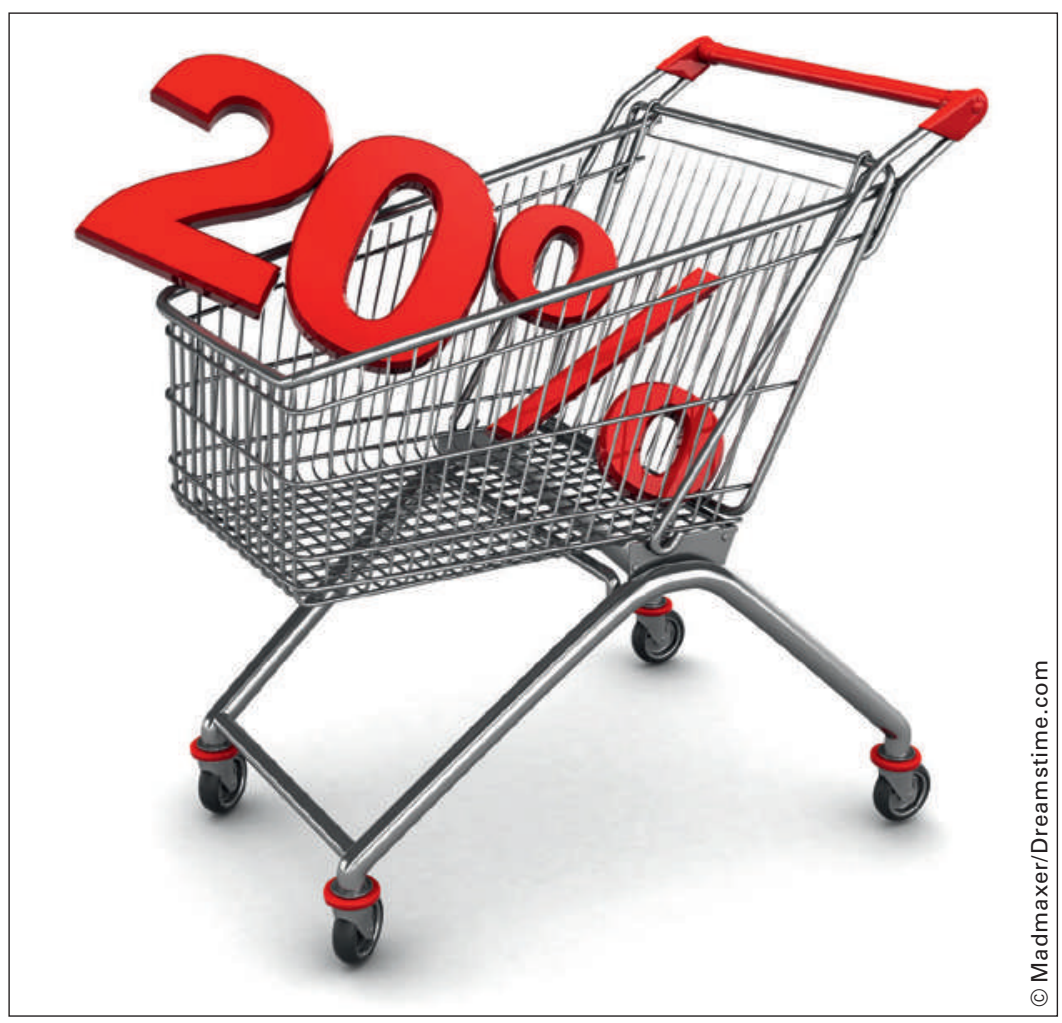

Mengenrabatte der Lieferanten dürfen nicht mit Abbau bei Schulungen, Support und Service einhergehen. und einer diaphysären Schaftverankerung in zwei fast gleich grosse Lager aufgeteilt. Die dritte, eher kleiner werdende Gruppe zementiert die Schäfte. Ähnliche "Fraktionen» und Glaubensrichtungen finden sich bei den Prothesenpfannen. Mit den verschiedenen Gleitpaarungen - Polyethylen, Metall, Keramik - ergeben sich bereits viele Varianten, Teilund Schnittmengen, die Mengenlehre lässt grüssen. Allen gemeinsam ist die Überzeugung, der höchsten Qualität verpflichtet zu sein - aber welcher Qualität?

\section{Unter dem ökonomischen Druck entscheidet immer mehr der Spitalträger über die Wahl der Implantate.}

Eines der wichtigsten Qualitätskriterien ist die Funktionsdauer der Implantate im menschlichen Körper. War der Goldstandard zu Beginn der Endoprothetik 10 Jahre, ist die sogenannte "Survival rate» heute deutlich höher. Doch wie weiss der "Kunde/Arzt» oder der "Endverbraucher/Patient», wie lange welches Produkt hält? Sicher ist, dass die Überlebensdauer der «Weltneuheit» noch unbekannt ist. Trotzdem verlangen die Patienten fast ausschliesslich das «Neueste» und nicht das Langbewährte. Viele Ärzte befriedigen diese Wünsche nach dem Neuesten wider besseres Wissen. Dabei wären Daten verfügbar. Seit Jahren geben verschiedene Implantatregister Auskunft über die Langzeitverläufe der Prothesen. Auch die Schweiz verfügt mit SIRIS - von Swiss Orthopaedics und dem Verband der Industrie FASMED gemeinsam gegründet - seit rund drei Jahren über ein Prothesenregister.

\section{Neu ist nicht immer besser}

Viele vollmundig angepriesene Neuheiten haben die Erwartungen nicht erfüllt. Im Gegenteil, eine grosse Anzahl Patienten sind Opfer von vorzeitigem Implantatversagen geworden: Re-Operationen, bleibende Schäden, oft eine deutliche Verschlechterung des Zustandes im Vergleich zu vor der Erstoperation. Einige Beispiele: zementierte Titanschäfte, MaterialBrüche von Steckverbindungen, Oberflächenprothesen, Metall-Metall-Gleitpaarungen, quietschende Keramik-Prothesen usw. Die Industrie und die Ärzteschaft wären gut beraten, Neuerung einer kritischeren Risiko-Abwägung zu unterziehen. Ist der $\mathrm{zu}$ erwartende Gewinn einer Neuheit gemessen am Goldstandard gering, sollten möglichst keine Risiken eingegangen werden. Innovation ja, Menschenversuche nein! Besonders zu denken geben jene jüngs- 


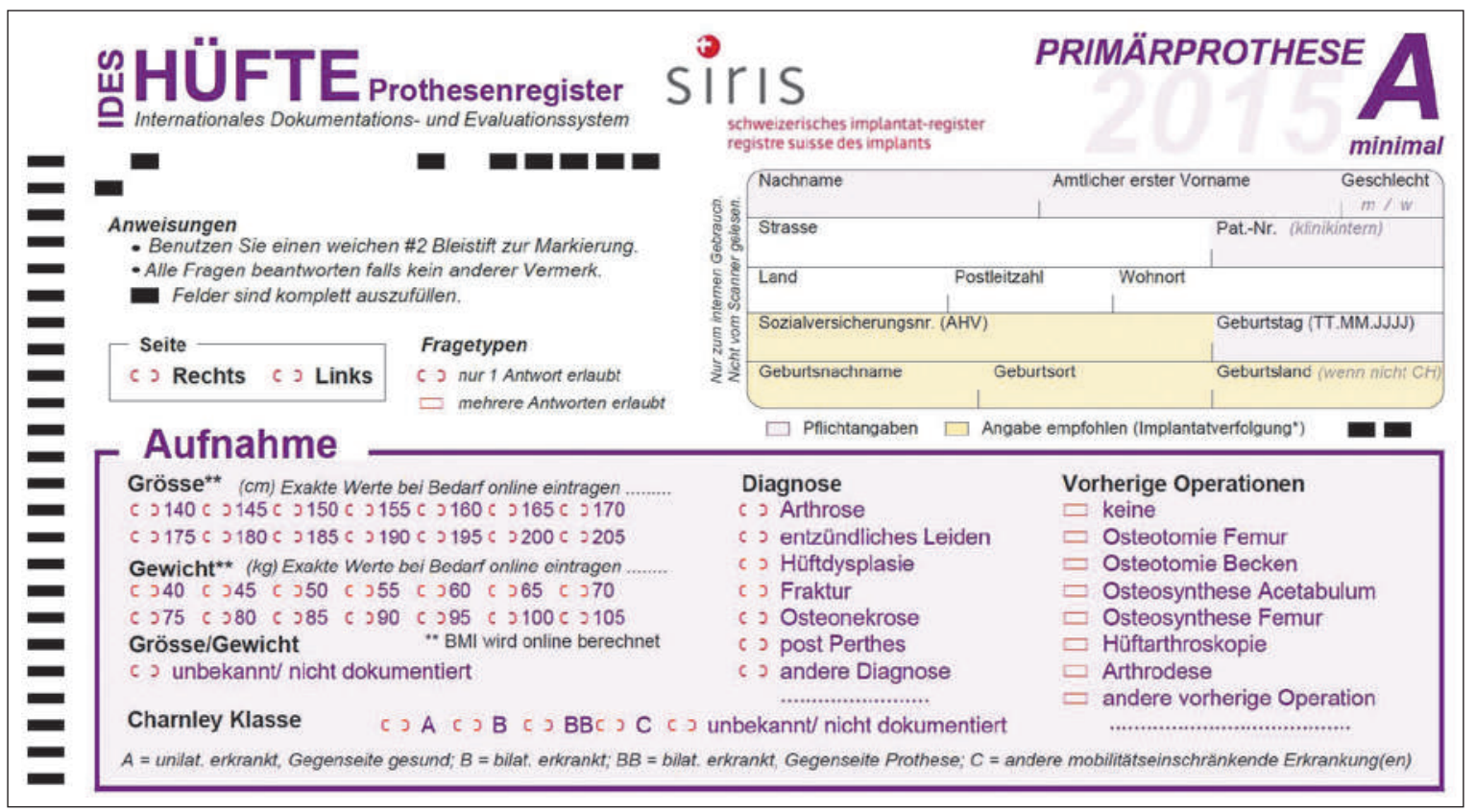

Qualitätserfassung zur Qualitätskontrolle: Seit 3 Jahren verfügt die Schweiz mit SIRIS über ein Prothesenregister.

ten «Innovationen», die bereits vor Jahren schon einmal gescheitert sind. Bei Kenntnis der medizinischen Entwicklung, der Biomechanik, der Naturgesetze überhaupt wäre eine Neuauflage des risikoreichen "Menschenversuchs» vermeidbar gewesen. Auch in der Medizin lernt man nichts aus der Geschichte. Und so werden wohl auch in Zukunft weitere Versuche mit Oberflächenprothesen der Hüfte oder Metall-Spacern bei Kniegelenken zu erwarten sein.

\section{Zusammenarbeit Ärzteschaft - Industrie}

Nachdem der Goldstandard in der Endoprothetik weitgehend erreicht ist, sind Verbesserungen fast nur noch bei den OP-Instrumenten zu erzielen. Viele Anbieter haben dies erkannt, arbeiten mit den Anwendern zusammen und setzen Tipps und Tricks der Praktiker um.

Viele vollmundig angepriesene Neuheiten haben die Erwartungen nicht erfüllt.

Dass letztere für diese Bemühungen vergütet werden, ist mehr als recht. Zuwendungen an Ärzte mit dem einzigen Zweck, diese zur Wahl eines bestimmten Produktes zu bewegen, sind unzulässig und grenzen an Korruption. Unter dem Druck der Behörden hat in den US-amerikanischen Hauptquartieren der Prothesenhersteller ein Umdenken stattgefunden. «Legal and Compliance» ist angesagt. Selbst der Einsatz von Firmenmitarbeitern, die im Operationssaal beim Zusammenbau von komplexen Revisionspro- thesen und Instrumenten Hilfe leisten, ist in den USA verboten, obwohl dies sinnvoll und qualitätsfördernd ist. Routinemässige Assistenzen von Aussendienstmitarbeitern bei Standardeingriffen - als nichtärztliche Assistenz abgerechnet und dem Operationshonorar zugeschlagen - sind definitiv illegal. Auch vor dem Hintergrund der Haftungsfrage sind solche Einsätze unzulässig.

\section{Implantatwahl und Revisionsoperation}

Die erfreulichen Langzeitresultate lassen die Indikation zur endoprothetischen Versorgung bei immer jüngeren Menschen stellen. Trotzdem nehmen wegen der steigenden Lebenserwartung und mit der $\mathrm{Zu}$ nahme der Prothesenträger notgedrungen auch die Revisionsoperationen zu. Materialverschleiss oder periprothetische Frakturen sind neben den Infekten die häufigsten Revisionsgründe. Nicht immer müssen alle Prothesenteile gewechselt werden. Der Patient soll dem kleinstmöglichen Eingriff unterzogen werden. Aber welche vor Jahrzehnten implantierten Produkte sind noch erhältlich? Welche neuen Bestandteile dürfen mit den alten kombiniert werden? Stehen - gerade bei periprothetischen Frakturen überbrückende Revisionsprothesen zur Verfügung? Und in welcher Dringlichkeit können diese geliefert werden? Alles Probleme, die in Notfallsituationen zu Unzeit gelöst werden müssen. Das Schicksal will es, dass solche Notfälle fast immer Wochenenden und Feiertage bevorzugen. Daher ist der Operateur im 
konkreten Einzelfall auf den verlässlichen Support der Firmen angewiesen. Darin zeigt sich die Stärke eines Lieferanten. Diese Verfügbarkeit rund um die Uhr, die ausreichende Lagerhaltung und das technische Know-how der Mitarbeiter sind Vorhalteleistungen, die ihren Preis haben.

\section{Implantatwahl und DRG}

Mit der Einführung von SwissDRG vor drei Jahren hat in der Schweiz ein Paradigma-Wechsel stattgefunden. Während im System der Einzelleistungsabrechnung die Kosten eines Implantates für Arzt und Spital eine untergeordnete Rolle spielten, zwingt die pauschale Vergütung das Spitalmanagement zum Sparen. Davon sind die Implantate nicht ausgenommen. Unter diesem ökonomischen Druck entscheidet immer mehr der Spitalträger über die Wahl der Implantate. Als Erstes tendieren einzelne Häuser - auch ganze Spitalketten - zur Vereinheitlichung und Straffung der Produktepalette. Damit winken Mengenrabatte. Die Lagerhaltung wird in den einzelnen Operationsabteilungen reduziert. Es lassen sich möglicherweise personelle Ressourcen einsparen.

\section{Übernimmt für all diese schädlichen Folgen der Spitalträger die Verantwortung?}

Die Gefahr besteht, dass die im Wettbewerb obsiegenden Lieferanten ihrerseits die Preissenkungen kompensieren. Es fährt der Lastwagen vors Spital und lädt Kisten mit günstig erstandenem Prothesenmaterial ab. Die Schulung der Mitarbeitenden, der Support bei Revisionsoperationen, die Instandhaltung der Spezialinstrumente usw. sind nicht mehr inklusive und werden - wenn überhaupt noch angeboten - separat in Rechnung gestellt. Unrentable Vorhalteleistungen über Wochenende und Festtage werden dem Rotstift zum Opfer fallen. Das Nachsehen haben die Patienten, die solange unbehandelt im Spital liegen bleiben, bis das notwendige Material eingetroffen ist. Oder der Operateur entscheidet sich für die suboptimale Variante, wechselt unnötigerweise die ganze Prothese oder behilft sich mit allenfalls nicht kompatiblen Prothesenteilen. So führt eine rein preisorientierte Einkaufspolitik zum Qualitätsverlust. Übernimmt für all diese schädlichen Folgen der Spitalträger die Verantwortung? Eine Frage, die im Belegarztspital relevant wird. Gehen Schäden infolge unzulänglicher Implantate zu Lasten der Ärzte- schaft, hat dies rasch Auswirkungen auf die Versicherungsprämien.

\section{Lernkurve und Qualität}

Ein nicht zu unterschätzendes Problem ist die Qualitätsminderung während einer Lernphase. Jede Umstellung auf ein neues Prothesensystem ist von einer «learning curve» mit vermehrten Komplikationen begleitet. Daher führt ein langer Verbleib beim gewohnten OP-System und bei denselben Implantaten zu einem konstanten Qualitätsniveau. Werden zukünftig rein kostenorientierte Einkaufschefs oder spitalübergreifende Einkaufsgesellschaften in kurzen Zeitabständen den Ärzten die jeweils gerade billigsten Implantate in den OP stellen, werden die Komplikationen zunehmen, die Qualität unweigerlich sinken. Auch hier stellt sich wieder die Frage der Haftung. Im zunehmenden juristischen Trend, die Organisationshaftung geltend zu machen, kann diese Sparpolitik zum Bumerang fürs Spital werden.

\section{Schlussfolgerungen}

Zweifellos gilt es, den Gegebenheiten mit dem zunehmenden Kostendruck Rechnung zu tragen. Unerwünschte Entwicklungen sollen vermieden werden. Dazu folgende Anregungen:

- Die Wahl der Implantate ist - schon aus haftungsrechtlichen Gründen - Aufgabe der Ärztinnen und Ärzte.

- Aufgabe der Spital-Organe sind der Einkauf, die Aufrechterhaltung des Nachschubs und die Verhandlungen betreffend Lieferkonditionen.

- Im Liefervertrag sind die Vorhalteleistungen der Implantate-Firmen zu definieren und diese der Ärzteschaft zur Kenntnis zu bringen: Lagerbewirtschaftung, Personal-Schulung, Support bei Revisionen usw.

- Auch seien die seit 2013 definierten Richtlinien «Zusammenarbeit Ärzteschaft und Industrie» der Schweizerischen Akademie der medizinischen Wissenschaften in Erinnerung gerufen, zu finden online unter www.samw.ch/de/Ethik/Richtlinien/ Aktuell-gueltige-Richtlinien.html

- Im Weiteren sei auf das Positionspapier von Swiss Orthopaedics «Wahl der Implantate unter SwissDRG» verwiesen, erhältlich bei der Geschäftsstelle von Swiss Orthopaedics. 